\title{
Putting RFMix and ADMIXTURE to the test in a complex admixed population
}

\author{
Caitlin Uren * (D, Eileen G. Hoal(D) and Marlo Möller(D)
}

\begin{abstract}
Background: Global and local ancestry inference in admixed human populations can be performed using computational tools implementing distinct algorithms. The development and resulting accuracy of these tools has been tested largely on populations with relatively straightforward admixture histories but little is known about how well they perform in more complex admixture scenarios.

Results: Using simulations, we show that RFMix outperforms ADMIXTURE in determining global ancestry proportions even in a complex 5-way admixed population, in addition to assigning local ancestry with an accuracy of $89 \%$. The ability of RFMix to determine global and local ancestry to a high degree of accuracy, particularly in admixed populations provides the opportunity for more accurate association analyses.

Conclusion: This study highlights the utility of the extension of computational tools to become more compatible to genetically structured populations, as well as the need to expand the sampling of diverse world-wide populations. This is particularly noteworthy as modern-day societies are becoming increasingly genetically complex and some genetic tools and commonly used ancestral populations are less appropriate. Based on these caveats and the results presented here, we suggest that RFMix be used for both global and local ancestry estimation in worldwide complex admixture scenarios particularly when including these estimates in association studies.
\end{abstract}

Keywords: South Africa, Local ancestry inference, Population genetics, RFMix, ADMIXTURE

\section{Background}

Admixture, the exchange of genetic material between distinct populations, is a hallmark of modern society - it can occur between closely or distantly related populations [1]. This exchange of genetic material leads to population structure; the pattern, timing and extent has been investigated in detail in a number of populations [1-3]. Such studies on southern African populations are particularly noteworthy as this area is postulated to be the geographical origin of modern humans [4]. Furthermore, it was the final destination of various human population migrations

\footnotetext{
* Correspondence: caitlinu@sun.ac.za

DSI-NRF Centre of Excellence for Biomedical Tuberculosis Research, South African Medical Research Council Centre for Tuberculosis Research, Division of Molecular Biology and Human Genetics, Faculty of Medicine and Health Sciences, Stellenbosch University, Room 4036, 4th Floor Education Building, Francie van Zijl Drive, Cape Town 8000, South Africa
}

that have resulted in a unique pattern of genetic diversity in the region $[3,5-8]$. Therefore, investigating population structure in modern southern African populations may reveal more about the area's rich history.

Correctly and efficiently determining ancestral proportions in an admixed population is possible by using computational and statistical algorithms that adapt to a variety of demographic scenarios [9-11]. The ability to determine the ancestral origin of a particular chromosomal region (or the overall admixture proportions) in an admixed individual has enabled the mapping of the origins of genetic risk factors in complex disease [12-14]. The majority of the computational and statistical tools used for global and local ancestry inference (GAI and LAI respectively) were however tested on and tailored to 2- and 3-way admixed populations (such as African Americans, Hispanics and Latino's). The extension to more complex admixed 
populations and the evaluation of the resulting accuracy has yet to be investigated.

As with most geographical regions, southern Africa houses a multitude of diverse human populations that all share in the migratory history in the area. One of the most unique populations in southern Africa is the South African Coloured (SAC) population (as termed in the South African census). The SAC population received ancestral contributions from 5 distinct populations; Bantuspeaking African $(\sim 30 \%)$, KhoeSan $(\sim 30 \%)$, European $(\sim 20 \%)$, East Asian $(\sim 10 \%)$ and South East Asian populations $(\sim 10 \%)[3,5-8]$. The admixture began approximately 15 generations ago and followed a continuous migration model [3]. The extent, mode and timing of admixture events is unique and creates a highly complex population with heterogenous ancestral haplotypes and linkage disequilibrium patterns.

The first step in a study design aimed at characterizing a relationship between ancestry and disease (such as genome-wide association studies and admixture mapping) is to understand the ancestral composition of the study population. Here we have set out to test the accuracy of global and local ancestry inference in one of the most complex admixed populations world-wide, using newly available dense genotyping data. A simulated 5-way admixed population is generated, and global and local ancestry estimates are compared to the true values to determine the accuracy of the computational algorithm.

\section{Results}

The aim of this study was to determine the accuracy of global and local ancestry inference (GAI and LAI respectively) in one of the most complex populations world-wide- putting it to the ultimate test. In order to do this, a highly complex 5-way admixed population was simulated. The GAI and LAI estimates were then compared to the true simulated data.

\section{GAI accuracy}

A 5-way admixed southern African population was simulated. The average ancestry proportions across these individuals were in line with what is seen in the real-world (Table 1) [3]. The simulations provided the basis with which the global ancestry proportions as calculated by ADMIXTURE [11] and RFMix [9] could be compared.

Supervised and unsupervised admixture analysis of the simulated dataset by ADMIXTURE and that performed by RFMix, confirmed that the simulated 5-way admixed population is highly heterogenous. Average ancestral proportions for both computational tools are given in Table 1. The comparisons across the 5 ancestries for each simulated individual are also depicted in Fig. 1. Root Mean Squared Errors (RMSE) were calculated for each comparison. As per the RMSE's, RFMix outperforms both ADMIXTURE runs (unsupervised and supervised) in correctly estimating admixture proportions in the 5-way admixed population, with the exception of KhoeSan ancestry where the accuracy is largely equal. Both ADMIXTURE runs over-estimates the Bantuspeaking African contribution and under-estimates the KhoeSan ancestral proportions. Similarly, the unsupervised ADMIXTURE run overestimates European ancestry and underestimates South East Asian ancestry.

\section{LAI accuracy}

Beyond global ancestry proportions, the simulation of a 5-way admixed population resulted in known local ancestry tracts, to which calls by a computational tool can be compared. The ancestral origin of each parental chromosomal region was determined using RFMix. RFMix has been shown to outperform other computational tools in the estimation of local ancestry in complex admixture scenarios [13]. The local ancestry calls by RFMix were compared to the "true" simulated ancestral origin of each region. To determine the robustness of RFMix when inaccurate admixture timing estimates are available, we selected 10, 15 and 20 generations as input for time since admixture. Although there were differences in the accuracy of RFMix when the time since admixture was varied, these differences were not significant (except for Bantu-speaking ancestry) and the direction of these differences varied for each ancestral population (Fig. 2). For this reason, a time since admixture in line with the simulated population was used for further analyses (15 generations).

Table 1 Average admixture proportions

\begin{tabular}{llllll}
\hline & $\begin{array}{l}\text { Previously Reported (Uren et al. } \\
\text { 2016) (\%) }\end{array}$ & Simulation (\%) & $\begin{array}{l}\text { ADMIXTURE } \\
\text { (unsupervised) (\%) }\end{array}$ & $\begin{array}{l}\text { ADMIXTURE } \\
\text { (supervised) }(\%)\end{array}$ & RFMix (\%) \\
\hline $\begin{array}{l}\text { Bantu-speaking } \\
\text { African }\end{array}$ & 32 & $26(95 \%$ Cl: $25-28)$ & $33(95 \%$ Cl: $32-35)$ & $31(95 \%$ Cl: $30-33)$ & $27(95 \%$ Cl: $26-30)$ \\
KhoeSan & 30 & $33(95 \%$ Cl: $31-36)$ & $25(95 \%$ Cl: $23-27)$ & $34(95 \%$ Cl: $31-37)$ & $33(95 \%$ Cl: $30-36)$ \\
European & 19 & $23(95 \%$ Cl: $21-25)$ & $26(95 \%$ Cl: $24-29)$ & $21(95 \%$ Cl: $19-24)$ & $22(95 \%$ Cl: $20-24)$ \\
East Asian & 7 & $6(95 \%$ Cl: $5-9)$ & $7(95 \%$ Cl: $5-9)$ & $6(95 \%$ Cl: $5-8)$ & $6(95 \%$ Cl: $5-9)$ \\
South East Asian & 12 & $12(95 \%$ Cl: $10-15)$ & $9(95 \%$ Cl: $8-12)$ & $8(95 \%$ Cl: $7-11)$ & $12(95 \%$ Cl: $10-14)$ \\
\hline
\end{tabular}




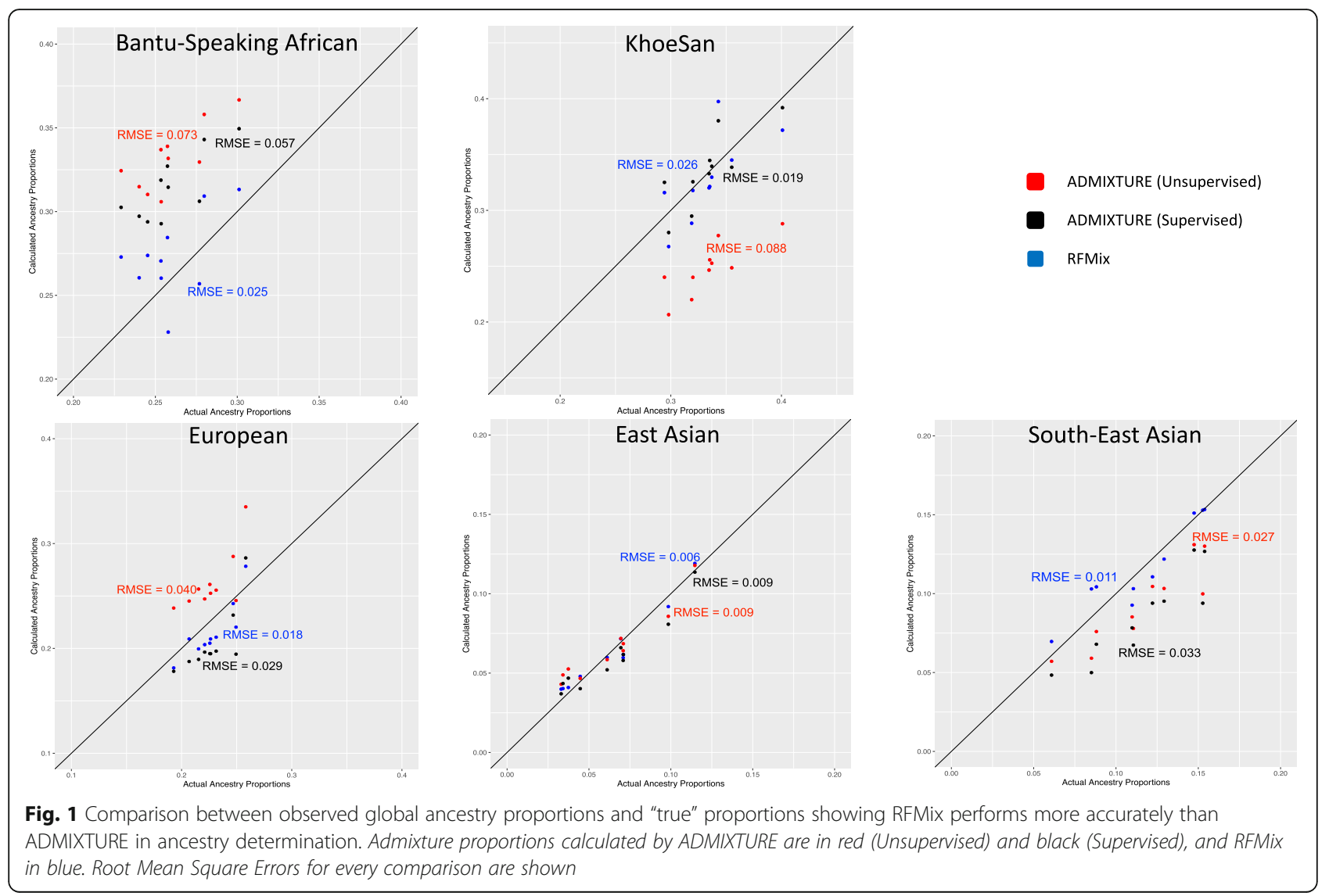

The overall LAI accuracy across all ancestries (15 generations) is $\sim 89 \%$; $88 \%$ accurate in calling Bantuspeaking African ancestry, 87\% calling KhoeSan ancestry, 95\% calling European ancestry, 86\% calling East Asian ancestry and 85\% calling South East Asian ancestry. The statistical significance of RFMix's ability to call a specific ancestry over another was assessed (Fig. 3). RFMix is able to call East Asian and European ancestry more precisely than any of the other ancestries (Fig. 3).

\section{Discussion}

From the results, we note that the estimates obtained for ADMIXTURE is greatly influenced by the inclusion of admixed reference populations. For example, the undercalling of South East Asian ancestry is most likely due to inherent European ancestry present in South East Asian populations and likewise, Bantu-speaking ancestry in the KhoeSan population. This is consistent with the trends seen in the LAI accuracy analysis. This highlights the need for further improvement in computational tools to distinguish between intra-continental ancestral populations, particularly in Africa as well as to perhaps tailor these tools to complex admixture scenerios where admixture with particular ancestral populations occurred at different times [9]. This is particularly noteworthy as most modern-day populations are admixed and therefore computational tools should be able to account for this within the algorithms.

The evident difference in accuracy estimating admixture proportions using RFMix and ADMIXTURE can be attributed to a number of aspects. RFMix is able to harness a multitude of prior information in order to perform LAI (and therefore GAI) such as LD, relatedness and phase information. Overall, we hypothesize that the addition of this information allows for the increased accuracy of RFMix over ADMIXTURE. Additionally, it is interesting to note that ADMIXTURE's unsupervised algorithm which is used to tease out fine-scale population structure in admixed populations, performed poorly in relation to the supervised algorithm. This is significant since it is the most widely used ADMIXTURE algorithm and highlights the necessity to move away from estimations not based on haplotypes.

We tested the robusteness of RFMix in calling local ancestry tracts when the incorrect time since admixture was used in the model. In our simulated dataset, we did not find any statistical differences when using an inflated or deflated time since admixture, with the excpetion of calling Bantu-speaking ancestry. This apparent decrease in accuracy using a time since admixture of 15 generations is largely a by-product of the increased accuracy seen when calling KhoeSan ancestry; similar to the trend 


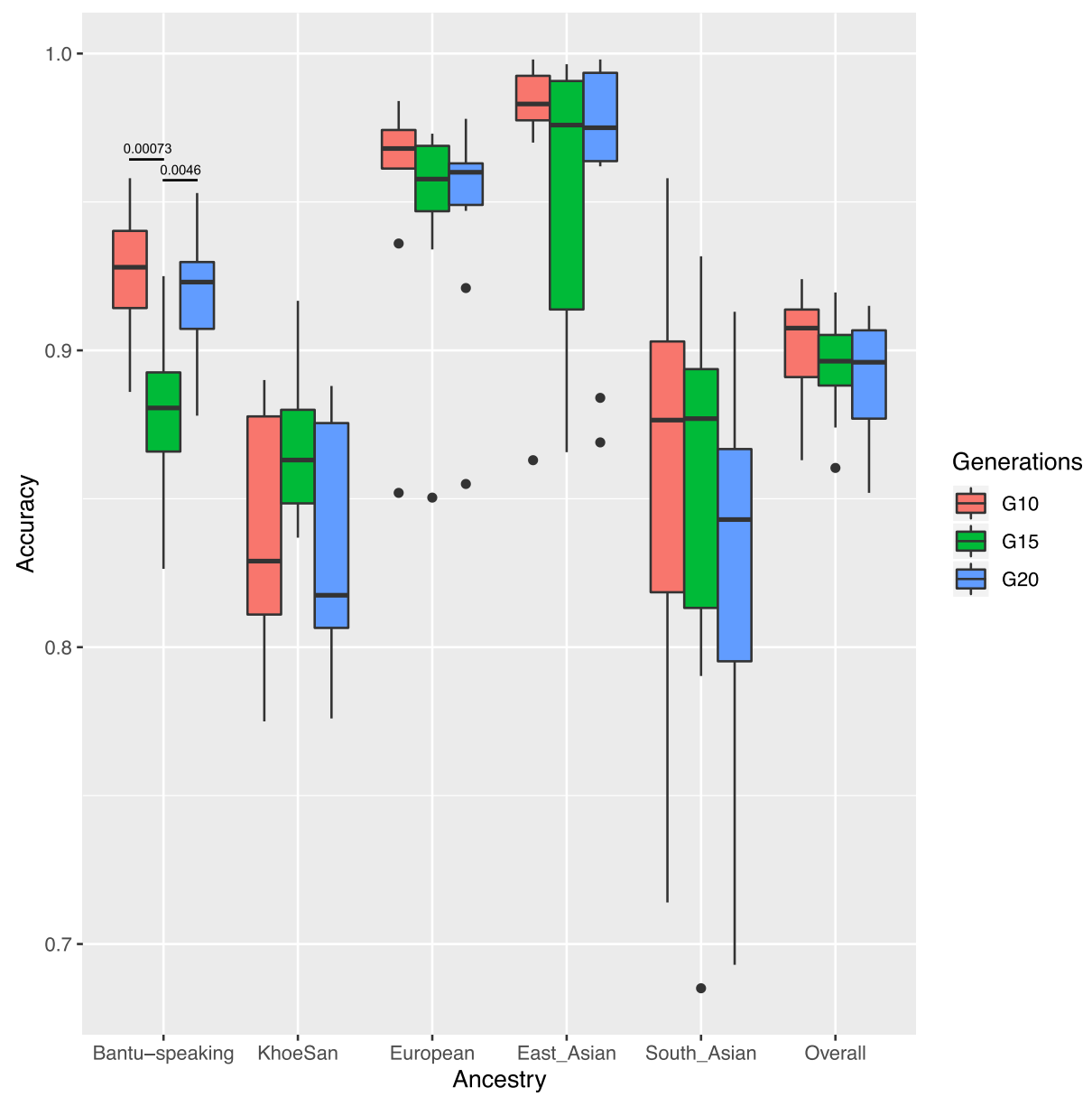

Fig. 2 Boxplot showing the robustness of RFMix when using inaccurate time since admixture estimates. Time since admixture of 10 (red), 15 (green) and 20 (blue) generations are shown. The median (bold horizontal line) and the upper and lower quartiles are shown. Data outside this range are plotted as outliers. The differences in accuracies across generations for each ancestry were assessed using a Wilcoxon non-parametric test. All statistically significant $p$ values $(<0.01)$ are shown

seen in the GAI accuracy results. Studies that under- or overestimate the time since admixture as well as include admixed ancestral populations that are genetically similar, may experience similar trends with LAI accuracy values across ancestral populations i.e. calling tracts of one ancestral population accurately might decrease the accuracy of another ancestral population that is closely related. One way to pre-empt this would be to incorporate (within the computational algorithm) the specification of a time since admixture for each ancestral population.

\section{Conclusion}

In conclusion, the findings presented here is the first of its kind to detail the accuracy of LAI and GAI in one of the most complex populations worldwide. Due to the accuracy and versatility of RFMix which harnesses prior information as LD, relatedness and phase information in determining global and local ancestry in a single program, it should be the algorithm of choice to characterize more complex admixture scenarios. The inclusion of accurate admixture proportions as a covariate in association studies is vital, and it is our opinion that researchers studying complex admixed populations should use RFMix for this purpose.

Furthermore, we demonstrate that computational tools are able to decipher the complex African genetic history with a high degree of accuracy, but there is still some room for improvement regarding the tailoring of computational tools to handle intra-continental, admixed reference and target populations.

As populations become increasingly mobile, the likelihood of admixture between diverse groups is greater. Therefore the extension of these and future computational tools to genetically complex populations from across the world is vital and. The conclusions of this study are therefore relevant and generalizable to other admixed populations. 


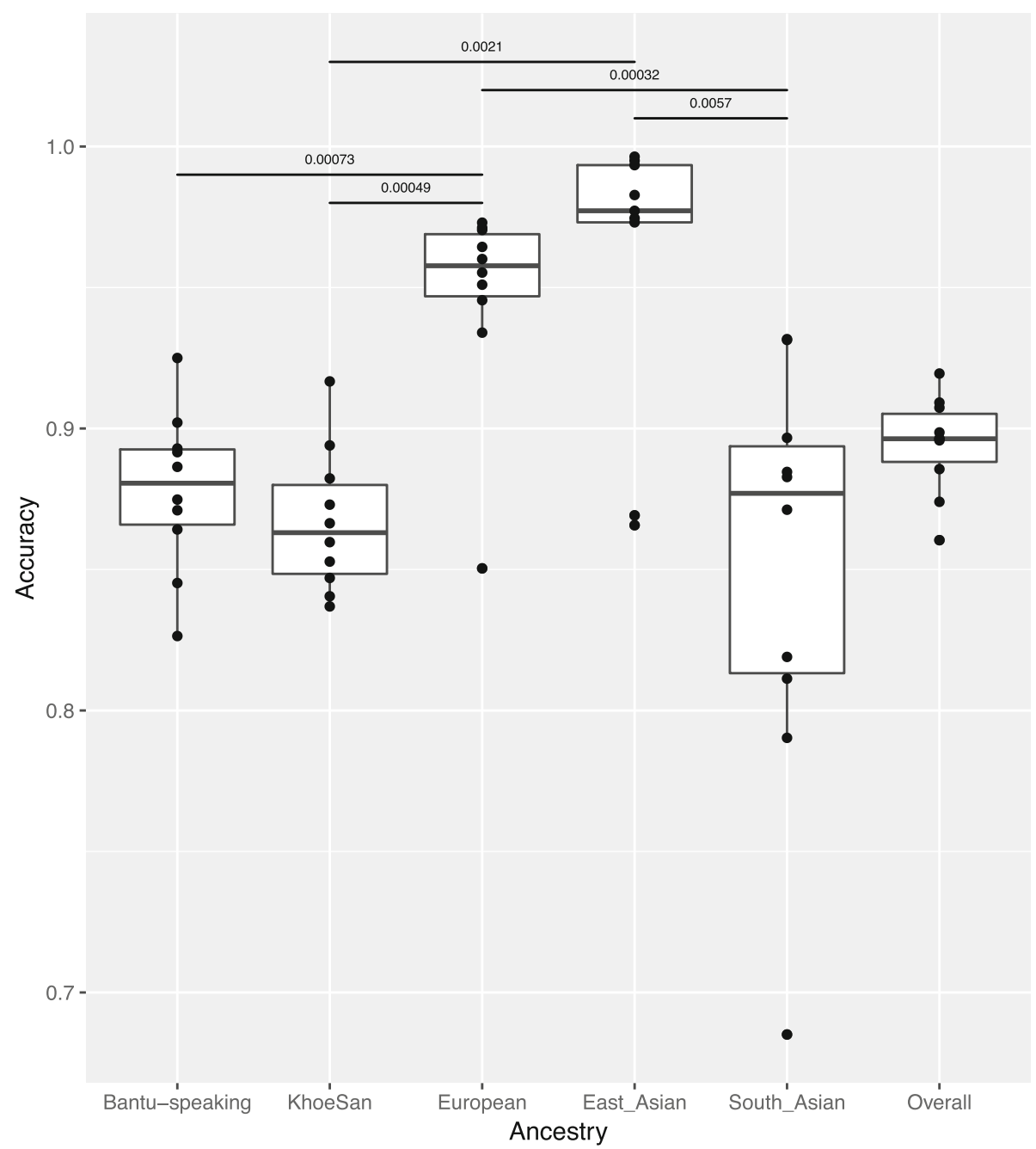

Fig. 3 Boxplot showing the accuracy with which RFMix assigns an ancestral origin to a genetic region, stratified by reference population. The median (bold horizontal line) and the upper and lower quartiles are shown. Data outside this range are plotted as outliers. The differences in accuracies across ancestries were assessed using a Wilcoxon non-parametric test. All statistically significant $p$ values $(<0.01)$ are shown

\section{Methods}

\section{Data merging and filtering}

KhoeSan genotype data from Martin and colleagues [15] was merged with the genetic data generated as part of the Population Architecture using Genomic and Epidemiology dataset [16] and genetic data from the Gujarati Indian and British populations from the 1000 Genomes Project [17]. Preliminary data filtering included a filter for minor allele frequency (0.003), missingness per genotype $(\max 0.05)$ and missingness per individual $(\max 0.01$ ). A total of $\sim 776 \mathrm{k}$ SNPs passed these filters and formed the initial merged dataset. Further data filtering is described in the appropriate sections below. Data was phased using SHAPEIT2 utilizing the published African American HapMap recombination map $[18,19]$. Populations in the final dataset are summarised in Table 2.

\section{Simulations}

The computational workflow is summarised in Fig. 4. A random subset of 55 reference individuals from the final merged dataset described in Table 2 was used to generate a simulated dataset using admix-simu (11 per reference population) [20]. The remaining 444 reference individuals formed the reference dataset for GAI and LAI. A demographic model consisting of specific ancestry proportions

Table 2 Population characteristics of the final merged dataset

\begin{tabular}{ll}
\hline Population & Number of individuals included \\
\hline KhoeSan (Nama and \#Khomani San) & 284 \\
European (British) & 79 \\
African (Yoruba and Luhya) & 35 \\
East Asian (Han) & 50 \\
South East Asian (Gujarati) & 103 \\
\hline
\end{tabular}




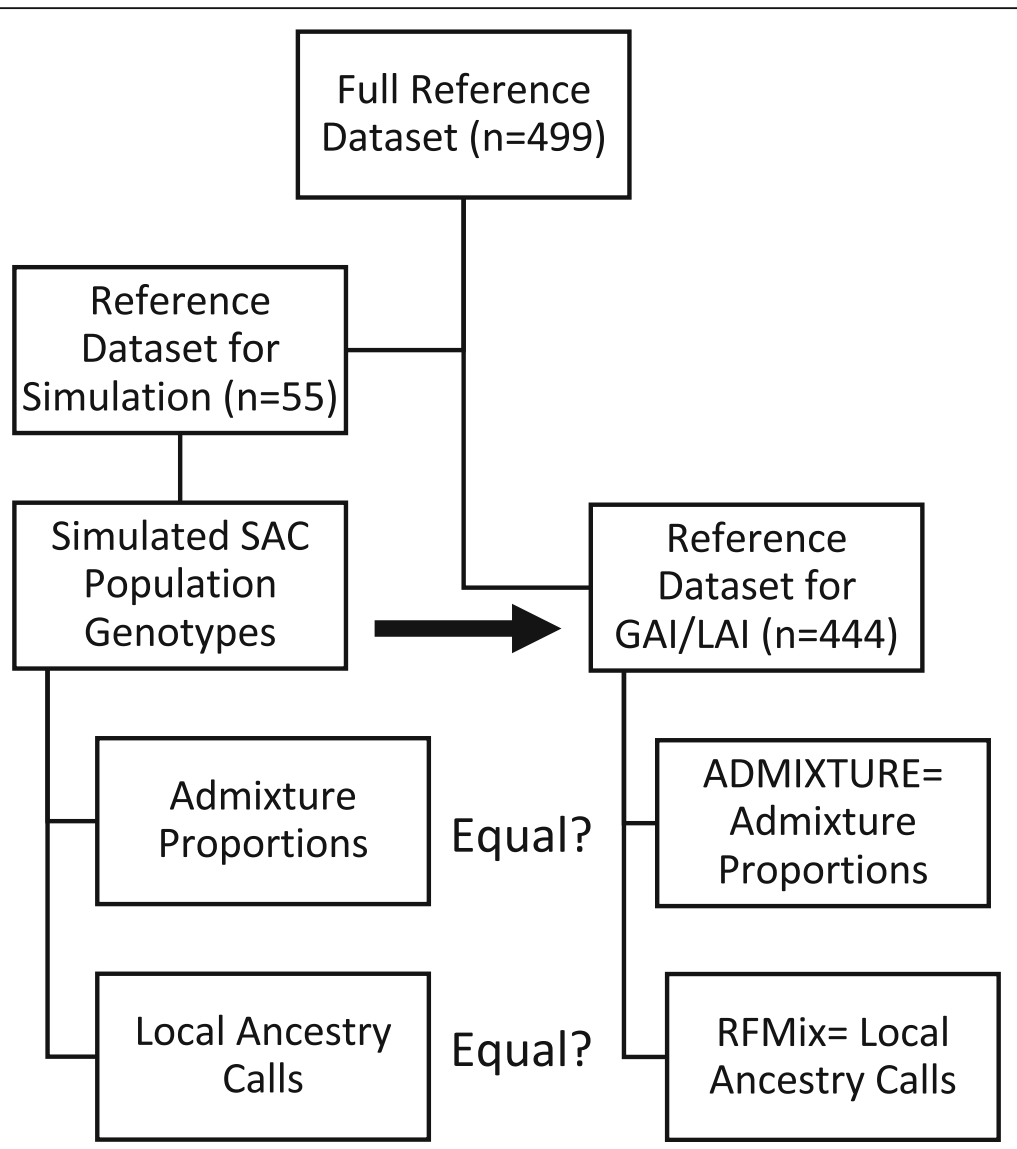

Fig. 4 Computational workflow. The full dataset $(n=499)$ was divided into a dataset used for the simulation $(n=55)$ and a dataset used for $G A 1$ and LAI $(n=444)$. Once the simulated SAC population was generated (including global and local ancestry estimations), these true values were compared to values emanating from ADMIXTURE and RFMix. For details, please see the methods section

and timing of migration, leading a continuous migration model initializing at 15 generations ago, was used to generate a simulated 5-way admixed population [3] (please see Table S1 for the specific admixing proportions). This simulation results in a heterogenous population, reminiscent of a real-world SAC population (see Table 1).

The simulation does not take post-admixture selection into account since it is highly unlikely that 350 years would result in distinct selection signals, rather, the inherent selection signals in the source populations will be transferred in a random manner to the simulated admixed population (adaptive introgression). Genotype as well as local ancestry calls were generated for this simulated dataset from real reference haplotypes, thus capturing the complexity of this heterogenous 5-way admixed South African population.

\section{Software choices}

Although there are a number of software programs that are able to estimate global ancestry (BAPS [21], HAPMIX [22], LAMP [23], FRAPPE [24], sNMF [25] etc), ADMIXTURE is however the most utilized. Reasons for this include the ability to include related individuals in one run and to generate accurate admixture proportions using relatively low-density SNP-array data [11]. The other widely used global ancestry algorithm, STRUCTURE has been shown to overestimate admixture proportions in even simple admixture scenarios, therefore given the demographic history of the population presented here, this software was not used [26].

RFMix was chosen as the local ancestry inference algorithm of choice as it allows for parameter optimization given the number of ancestral populations and the ability to perform LAI in populations more than 2-way or 3way admixed (limitations of LAMP [23] and HAPMIX [22]). In addition, RFMix has the inherent ability to calculate local and global ancestry simultaneously and allows for array-based input data as well as whole genome sequencing data. Furthermore, initial results by Daya and colleagues suggested that RFMix is the most accurate tool for local ancestry estimation (over and above that calculated for LAMP-LD $[27,28]$ ) in admixed southern African populations however, only a 3-way admixture scenario was tested (San, Bantu-speaking and non-African) [13]. 


\section{GAl accuracy}

Reference individuals not included in the dataset used for the simulation, were allocated to the dataset used for GAI and LAI. Global ancestry proportions were determined by ADMIXTURE [11] and RFMix [9]

The ADMIXTURE analysis was performed in a supervised and unsupervised manner after filtering the dataset for linkage disequilibrium as per the manual's recommendations (50 kb window size, step size of $10 \mathrm{~kb}$ and $R^{2}$ threshold of 0.1). The supervised algorithm allows for the input of know ancestral origins of the reference individuals whereas the unsupervised algorithm infers the ancestry of all individuals given as input.

RFMix was run using default parameters, a time since admixture of 15 generations (in line with the simulation) as well as 3 expectation-maximization (EM) iterations (further EM iterations were not shown to increase accuracy [9]). The correlation of the two methods by means of the Root Mean Squared Error (RMSE) was performed in R.

\section{LAI accuracy}

Local ancestry calls were generated by RFMix using the same parameters as described in the previous section. The ability to correctly assign local ancestry was calculated in two ways, at an individual level. The first determined the global accuracy using the formula $\frac{d_{c}}{d_{t}}$, where $d_{c}$ is the number of sites that had a called ancestry and $d_{t}$ is the number of sites that had a correctly called ancestry as compared to the simulations. The second method of accuracy estimation looked at this accuracy per ancestral population using the formula $\frac{d_{c a}}{d_{a}}$ where $d_{a}$ is the number of sites that had a called ancestry and $d_{c a}$ is the number of sites that the specific ancestry was correctly called [29]. These accuracy estimators were then averaged over all individuals in the simulated 5-way admixed dataset.

\section{Supplementary information}

Supplementary information accompanies this paper at https://doi.org/10. 1186/s12863-020-00845-3.

Additional file 1: Table S1. Demographic model used to simulated SAC population

\section{Abbreviations}

GAl: Global ancestry inference; LAl: Local ancestry inference; SAC: South African coloured; SNP: Single nucleotide polymorphism; LAMP: Local ancestry in admixed populations; LD: Linkage disequilibrium; kb: Kilobase;

EM: Expectation maximization; RMSE: Root mean square error

\section{Acknowledgements}

We thank Dr's Brenna Henn, Elizabeth Atkinson and Chris Gignoux for their assistance and support of this research. We thank the study participants of the projects cited here - without their contribution, this research would not be possible.

\section{Authors' contributions}

CU designed the study, wrote the first draft of the manuscript and performed the computational analyses. MM and EH helped to develop the research and edited the manuscript. All authors have read and approved this manuscript.

\section{Funding}

This research was funded (partially or fully) by the South African government through the South African Medical Research Council and the National Research Foundation. CU was supported by a fellowship from the Claude Leon Foundation. The funders had no role in the design or implementation of this study or the drafting of the manuscript.

\section{Availability of data and materials}

No new genetic data was generated for this study however all reference data supporting the findings of this study are available via the original publication. The 1000 Genomes data can be access accessed by visiting https://www.internationalgenome.org. The European Nucleotide Archive accession number for the 1000 Genomes data is PRJNA262923. The Population Architecture using Genomics and Epidemiology (PAGE) dataset can be found on dbGap with accession number phs000356.v2.p1. Genotyping data for the KhoeSan population can be accessed on application to the Stellenbosch University Health Research Ethics Committee with reference to study N11/07/210.

\section{Ethics approval and consent to participation}

All procedures performed in studies involving human participants were in accordance with the ethical standards of the institutional and/or national research committee and with the 1964 Helsinki declaration and its later amendments or comparable ethical standards. No new genetic data was collected/generated by this study however, all data utilized in this study has been provided by the respective study participants with written and/or verbal consent. This study was approved by the Stellenbosch University Health Research Ethics Committee (Reference N95/072, N06/07/132 and S17/ 01/013)

\section{Consent for publication}

Not Applicable.

\section{Competing interests}

The authors declare that they have no competing interests exist.

Received: 12 September 2019 Accepted: 24 March 2020

Published online: 07 April 2020

\section{References}

1. 1000 Genomes Project Consortium, Abecasis GR, Auton A, Brooks LD, DePristo MA, Durbin RM, et al. An integrated map of genetic variation from 1,092 human genomes. Nature. 2012;491(7422):56-65.

2. Gurdasani D, Carstensen T, Tekola-Ayele F, Pagani L, Tachmazidou I, Hatzikotoulas K, et al. The African genome variation project shapes medical genetics in Africa. Nature. 2015;517(7534):327-32.

3. Uren C, Kim M, Martin AR, Bobo D, Gignoux CR, van Helden PD, et al. Finescale human population structure in southern Africa reflects Ecogeographic boundaries. Genetics. 2016;204(1):303-14.

4. Henn BM, Gignoux CR, Jobin M, Granka JM, Macpherson JM, Kidd JM, et al. Hunter-gatherer genomic diversity suggests a southern African origin for modern humans. Proc Natl Acad Sci U S A. 2011;108(13):5154-62.

5. Petersen DC, Libiger O, Tindall EA, Hardie R-A, Hannick LI, Glashoff RH, et al. Complex patterns of genomic admixture within southern Africa. PLoS Genet. 2013;9(3):e1003309.

6. de Wit E, Delport W, Rugamika CE, Meintjes A, Moller M, van Helden PD, et al. Genome-wide analysis of the structure of the south African Coloured population in the Western cape. HumGenet. 2010;128(2):145-53.

7. Chimusa ER, Daya M, Möller M, Ramesar R, Henn BM, van Helden PD, et al. Determining ancestry proportions in complex admixture scenarios in South Africa using a novel proxy ancestry selection method. PLoS One. 2013;8(9): e73971.

8. Daya M, van der Merwe L, Galal U, Möller M, Salie M, Chimusa ER, et al. A panel of ancestry informative markers for the complex five-way admixed south African Coloured population. PLoS One. 2013 Dec 20;8(12):e82224. 
9. Maples BK, Gravel S, Kenny EE, Bustamante CD. RFMix: a discriminative modeling approach for rapid and robust local-ancestry inference. Am J Hum Genet. 2013 Aug 8;93(2):278-88.

10. Brown $R$, Pasaniuc B. Enhanced methods for local ancestry assignment in sequenced admixed individuals. PLoS Comput Biol. 2014;10(4):e1003555.

11. Alexander DH, Novembre J, Lange K. Fast model-based estimation of ancestry in unrelated individuals. Genome Res. 2009;19(9):1655-64.

12. Cheng CY, Kao WH, Patterson N, Tandon A, Haiman CA, Harris TB, et al. Admixture mapping of 15,280 African Americans identifies obesity susceptibility loci on chromosomes 5 and X. PLoSGenet. 2009;5(5):e1000490.

13. Daya $M$, van der Merwe L, Gignoux CR, van Helden PD, Möller M, Hoal EG. Using multi-way admixture mapping to elucidate TB susceptibility in the south African Coloured population. BMC Genomics. 2014;15:1021.

14. Freedman ML, Haiman CA, Patterson N, McDonald GJ, Tandon A, Waliszewska A, et al. Admixture mapping identifies $8 \mathrm{q} 24$ as a prostate cancer risk locus in African-American men. ProcNat|AcadSciUSA. 2006; 103(38):14068-73.

15. Martin AR, Lin M, Granka JM, Myrick JW, Liu X, Sockell A, et al. An Unexpectedly Complex Architecture for Skin Pigmentation in Africans. Cell. 2017;171(6):1340-1353.e14

16. Wojcik G, Graff M, Nishimura KK, Tao R, Haessler J, Gignoux CR, et al. The PAGE Study: How Genetic Diversity Improves Our Understanding of the Architecture of Complex Traits. bioRxiv. 2018;17:188094.

17. 1000 Genomes Project Consortium (2010). A map of human genome variation from population-scale sequencing. Nature. 2010;467(7319):1061-73.

18. O'Connell J, Gurdasani D, Delaneau O, Pirastu N, Ulivi S, Cocca M, et al. A general approach for haplotype phasing across the full Spectrum of relatedness. PLoS Genet. 2014;10(4):e1004234.

19. The International HapMap Consortium. A second generation human haplotype map of over 3.1 million SNPs. Nature. 2007:449(7164):851-61.

20. Williams A. admix-simu: admix-simu: program to simulate admixture between multiple populations [Internet]. 2016. Available from. https://doi. org/10.5281/zenodo.45517.

21. Corander J, Marttinen P, Sirén J, Tang J. Enhanced Bayesian modelling in BAPS software for learning genetic structures of populations. BMC Bioinformatics. 2008;9:539.

22. Price AL, Tandon A, Patterson N, Barnes KC, Rafaels N, Ruczinski I, et al. Sensitive detection of chromosomal segments of distinct ancestry in admixed populations. PLoS Genet. 2009;5(6):e1000519.

23. Sankararaman S, Sridhar S, Kimmel G, Halperin E. Estimating local ancestry in admixed populations. Am J Hum Genet. 2008:82(2):290-303.

24. Tang H, Peng J, Wang P, Risch NJ. Estimation of individual admixture: analytical and study design considerations. Genet Epidemiol. 2005;28(4):289-301.

25. Frichot $\mathrm{E}$, Mathieu F, Trouillon T, Bouchard G, François O. Fast and efficient estimation of individual ancestry coefficients. Genetics. 2014;196(4):973-83.

26. Cheng JY, Mailund T, Nielsen R. Fast admixture analysis and population tree estimation for SNP and NGS data. Bioinformatics. 2017;33(14):2148-55.

27. Baran Y, Pasaniuc B, Sankararaman S, Torgerson DG, Gignoux C, Eng C, et al. Fast and accurate inference of local ancestry in Latino populations. Bioinformatics. 2012;28(10):1359-67.

28. Chimusa ER, Zaitlen N, Daya M, Möller M, van Helden PD, Mulder NJ, et al. Genome-wide association study of ancestry-specific TB risk in the south African Coloured population. Hum Mol Genet. 2014;23(3):796-809.

29. Atkinson E. Calculations of accuracy comparing Williams lab simulations to RFmix runs: eatkinson/LAlaccuracy [Internet]. 2018 [cited 2019 Feb 12]. Available from: https://github.com/eatkinson/LAlaccuracy.

\section{Publisher's Note}

Springer Nature remains neutral with regard to jurisdictional claims in published maps and institutional affiliations.

Ready to submit your research? Choose BMC and benefit from:

- fast, convenient online submission

- thorough peer review by experienced researchers in your field

- rapid publication on acceptance

- support for research data, including large and complex data types

- gold Open Access which fosters wider collaboration and increased citations

- maximum visibility for your research: over $100 \mathrm{M}$ website views per year

At BMC, research is always in progress.

Learn more biomedcentral.com/submissions 